\title{
APPLICATION OF THE WEIGHTED-SUM-OF-GRAY-GASES MODEL TO NON-HOMOGENEOUS H2O/CO2 MIXTURES FOR MEDIA WITH NON-GRAY WALLS
}

\author{
R. J. C. da Fonseca, \\ and F. H. R. França \\ Universidade Federal do Rio Grande do Sul \\ Departamento de Engenharia Mecânica \\ Escola de Engenharia \\ Rua Sarmento Leite, 425 \\ Bairro Centro Histórico \\ Porto Alegre, Rio Grande do Sul, Brasil \\ roberta.fonseca@ufrgs.br \\ ABSTRACT \\ The spectral modeling of the thermal radiation in participating media is a \\ research area that has received constant attention due to its importance in a \\ great number of engineering problems, especially because the highly \\ irregular spectral dependence of the absorption coefficient of a gas with the \\ wavenumber requires the adoption of alternative methods to determine the \\ radiative properties. This work brings a numerical study of a one- \\ dimensional system, bounded by perfectly diffuse and non-gray walls, filled \\ by a non-homogeneous and non-isothermal mixture of water vapor and \\ carbon dioxide. The main objective is to estimate the magnitude of the \\ deviations in treating surfaces that should be considered non-gray as black \\ or gray. The spectral modeling of the problem is performed by the \\ weighted-sum-of-gray-gases (WSGG) model and the accuracy of the \\ solution methodology developed is evaluated by means of comparisons \\ against the results obtained by line-by-line (LBL) integration. \\ Received: April 16, 2018 \\ Revised: May 10, 2018 \\ Accepted: June 20, 2018 \\ Keywords: thermal radiation; weighted-sum-of-gray-gases model; line-by- \\ line integration; non-gray walls
}

\section{NOMENCLATURE}

temperature-dependent coefficient related to the $\mathrm{j}$-th gray gas, dimensionless

$\mathrm{a}_{\mathrm{jk}}$

fraction of the blackbody emission from the band interval $\Delta \eta_{\mathrm{k}}$, dimensionless

$\mathrm{a}_{0}$ temperature coefficient for the transparent windows, dimensionless

$b_{j, k} \quad$ polynomial coefficients of the $\mathrm{k}$-th order for the $\mathrm{j}$-th gray gas, varying units

$\mathrm{I}_{\mathrm{b}} \quad$ total blackbody intensity, $\mathrm{W} /\left(\mathrm{m}^{2} \mathrm{sr}\right)$

$\mathrm{I}_{\eta} \quad$ spectral radiative intensity, $\mathrm{W} /\left(\mathrm{m}^{2} \mathrm{~cm}^{-1} \mathrm{sr}\right)$

$\mathrm{I}_{\eta \mathrm{b}} \quad$ blackbody spectral intensity, $\mathrm{W} /\left(\mathrm{m}^{2} \mathrm{~cm}^{-1} \mathrm{sr}\right)$

$\mathrm{I}_{\mathrm{jk}, 1}^{+}$

partial intensity in the positive direction, $\mathrm{W} /\left(\mathrm{m}^{2} \mathrm{sr}\right)$

$\mathrm{I}_{\mathrm{jk}, 1}^{-}$

partial intensity in the negative direction, $\mathrm{W} /\left(\mathrm{m}^{2} \mathrm{sr}\right)$

$\mathrm{I}_{\eta, 1}^{+} \quad$ positive spectral intensity, $\mathrm{W} /\left(\mathrm{m}^{2} \mathrm{~cm}^{-1} \mathrm{sr}\right)$

$\mathrm{I}_{\eta, 1}^{-} \quad$ negative spectral intensity, $\mathrm{W} /\left(\mathrm{m}^{2} \mathrm{~cm}^{-1} \mathrm{sr}\right)$

$\mathrm{p} \quad$ total pressure of the system, atm

$\mathrm{p}_{\mathrm{a}} \quad$ partial pressure of participating species, atm

$\mathrm{q}_{\mathrm{R}}^{\prime \prime}$ radiative heat flux, $\mathrm{W} / \mathrm{m}^{2}$

$\dot{\mathrm{q}}_{\mathrm{R}}$ radiative heat source, $\mathrm{W} / \mathrm{m}^{3}$

$\mathrm{S}$ position in the path, $\mathrm{m}$

$\mathrm{T}$ temperature, $\mathrm{K}$

$\mathrm{X} \quad$ position along of the domain, $\mathrm{m}$

Y molar concentration, dimensionless

\section{Greek symbols}

$\alpha \quad$ wall absorptivity, dimensionless

$\delta \quad$ normalized deviation of the radiative heat

flux, \%

$\Delta \eta_{\mathrm{k}} \quad$ spectral band interval, $\mathrm{cm}^{-1}$

$\zeta$ normalized deviation of the radiative heat source, $\%$

$\varepsilon \quad$ wall hemispherical total emissivity, dimensionless

$\varepsilon_{\eta} \quad$ wall hemispherical spectral emissivity, dimensionless

$\eta \quad$ wavenumber, $\mathrm{cm}^{-1}$

$\theta \quad$ angle between the solid angle $\omega$ and the normal vector to the wall element, rad

$\kappa_{\eta} \quad$ medium spectral absorption coefficient, $\mathrm{m}^{-1}$

$\kappa_{\mathrm{pj}} \quad$ pressure absorption coefficient for the $\mathrm{j}$-th gray gas, $(\mathrm{m} \text { atm })^{-1}$

$\lambda \quad$ wavelength, $\mu \mathrm{m}$

$\mu_{1} \quad$ directional cosine, dimensionless

$\omega \quad$ solid angle, $\mathrm{sr}$

$\omega_{1} \quad$ quadrature weight for 1 direction, dimensionless

\section{Subscripts}

avg average

$\mathrm{CO}_{2}$ carbon dioxide

$\mathrm{H}_{2} \mathrm{O}$ water vapor

max maximum

w wall

\section{INTRODUCTION}

Thermal radiation is a very important mechanism of heat transfer in high-temperature 
processes involving participating species, such as water vapor and carbon dioxide. Line-by-line (LBL) integration, although capable of providing the most reliable modeling of the spectral behavior of these participating gases, is often impracticable due to the elevated computational effort required to account for the thousands of millions of spectral lines that compose the absorption coefficient of the medium. Alternatively, some simpler spectral models, as the weighted-sum-of-gray-gases (WSGG) model, have been capable of obtaining satisfactory agreement with results of LBL benchmark solution (Dorigon et al., 2013; Cassol et al., 2014; Brittes et al., 2017). However, spectral models are traditionally studied for problems bounded by black surfaces, although recently the cumulative wavenumber $(\mathrm{CW})$ model was extended for non-gray walls (Solovjov et al., 2013). In the solution, the wall spectral emissivity was represented by a few stepwise values in wide bands that were synchronized with the bands used in the discretization of the $\mathrm{CW}$ distribution function. Thus, the radiative transfer equation (RTE) was integrated in each band for the solution of the fractional gray gas intensity and the results present in the paper were generated for a 1D-medium slab with uniform concentrations of $\mathrm{H}_{2} \mathrm{O}$. Regarding to the application of the WSGG model to non-black surfaces, the literature relies on the work of Fonseca et al., 2015, for gray walls, and with the studies of Fonseca and França (2016), and Fonseca (2017), for non-gray walls, all considering homogeneous mixtures of water vapor and carbon dioxide.

In the present paper, the WSGG model is applied to solve the radiative heat transfer in a onedimensional system formed by two infinite flat parallel plates filled by a non-homogeneous mixture of $\mathrm{H}_{2} \mathrm{O}$ and $\mathrm{CO}_{2}$, for different non-isothermal temperature profiles. The solution methodology employed is the same that was applied in Fonseca (2017), but with emphasis in problems in which the molar concentrations vary throughout the domain. The main objective of this work is to compare previous results of the literature, obtained for black surfaces, with solutions generated assuming non-gray boundaries. Additionally, results implementing the WSGG model to non-gray and gray surfaces-bounded problems are compared with line-by-line integration applied to problems with non-gray walls, in order to quantify the discrepancy in modeling the boundaries as black when they are not. All simulations are performed using an in-house Fortran code, in which the spatial integration of the RTE was solved by the discrete ordinate method (DOM) considering 30 directions.

\section{PHYSICAL AND MATHEMATICAL MODELING}

\author{
The Radiative Transfer Equation and the LBL \\ Integration
}

Assuming a participating and non-scattering medium, the spectral radiative transfer equation (RTE) along a certain path $\mathrm{s}$ is given by (Siegel and Howell, 2002; Modest, 2003):

$$
\frac{\mathrm{dI}_{\eta}}{\mathrm{ds}}=-\kappa_{\eta}(\mathrm{s}) \mathrm{I}_{\eta}(\mathrm{s})+\kappa_{\eta}(\mathrm{s}) \mathrm{I}_{\eta \mathrm{b}}(\mathrm{s})
$$

in which $\kappa_{\eta}$ is the medium spectral absorption coefficient, in $\mathrm{m}^{-1}$, associated to the wavenumber $\eta$; $\mathrm{I}_{\eta}$ indicates the spectral radiation intensity, in $\mathrm{W} /\left(\mathrm{m}^{2} \mathrm{~cm}^{-1}\right)$; and $\mathrm{I}_{\eta \mathrm{b}}$ represents the blackbody spectral intensity at position $\mathrm{s}$ in the path, also in $\mathrm{W} /\left(\mathrm{m}^{2} \mathrm{~cm}^{-1}\right)$. To solve the Eq. (1), is required the definition of one boundary condition, so that, considering a perfectly diffuse surface, the spectral radiation intensity at the wall boundary in $\mathrm{s}=0$ can be calculated according to:

$$
I_{\eta}(s=0)=\varepsilon_{\eta} I_{\eta b}(s=0)+\frac{\left(1-\varepsilon_{\eta}\right)}{\pi} \int_{2 \pi} I_{\eta, i} \cos \theta d \omega
$$

where $\varepsilon_{\eta}$ is wall hemispheric spectral emissivity; $\mathrm{I}_{\eta, \mathrm{i}}$ is the incident spectral intensity on the wall element within the solid angle $\omega$; and $\theta$ is the angle between $\omega$ and the normal vector to the wall element.

In the present work, the spectral integration of the RTE is solved for a one-dimensional medium slab bounded by two infinite parallel plates, as shown in Fig. 1. The directional integration is performed with the discrete ordinates method (DOM), in which the RTE is solved for a set of directions and is used a Gauss-Legendre quadrature to determine the continuous integral over the solid angle. For a given direction 1, the RTE presented in Eq. (1) can be rewritten for the positive and negative spectral intensities, $\mathrm{I}_{\eta 1}^{+}$and $\mathrm{I}_{\eta 1}^{-}$, respectively:

$$
\begin{gathered}
\mu_{1} \frac{d I_{\eta, 1}^{+}}{d x}=-\kappa_{\eta}(x) I_{\eta, 1}^{+}(x)+\kappa_{\eta}(x) I_{\eta b}(x) \\
-\mu_{1} \frac{d I_{\eta, 1}^{-}}{d x}=-\kappa_{\eta}(x) I_{\eta, 1}^{-}(x)+\kappa_{\eta}(x) I_{\eta b}(x)
\end{gathered}
$$

in which $\mu_{1}$ is the directional cosine, as shown in Fig. 1. The boundary conditions for the walls to the left ( $\mathrm{x}$ $=0)$ and to the right $(\mathrm{x}=\mathrm{X})$ of the domain are given, respectively, by:

$$
\begin{aligned}
& \mathrm{I}_{\eta, 1}^{+}(\mathrm{x}=0)=\varepsilon_{\eta} \mathrm{I}_{\eta \mathrm{b}}(\mathrm{x}=0) \\
& +\frac{\left(1-\varepsilon_{\eta}\right)}{\pi} \sum_{1=1}^{\mathrm{L}} 2 \pi \mu_{1} \omega_{1} \mathrm{I}_{\eta, 1}^{-}(\mathrm{x}=0) \\
& \mathrm{I}_{\eta, 1}^{-}(\mathrm{x}=\mathrm{X})=\varepsilon_{\eta} \mathrm{I}_{\eta \mathrm{b}}(\mathrm{x}=\mathrm{X})
\end{aligned}
$$




$$
+\frac{\left(1-\varepsilon_{\eta}\right)}{\pi} \sum_{\mathrm{l}=1}^{\mathrm{L}} 2 \pi \mu_{1} \omega_{1} \mathrm{I}_{\eta, \mathrm{l}}^{+}(\mathrm{x}=\mathrm{X})
$$

where $\mathrm{L}$ is the number of discrete directions. For simplicity, in the equations it is assumed that the two surfaces present the same spectral distribution of emissivity, although the methodology developed is not restricted to this particular case.

Solving Eqs. (5) and (6) for all directions and integrating spectrally, the radiative heat flux, $\mathrm{q}_{\mathrm{R}}$, in $\mathrm{W} / \mathrm{m}^{2}$, and the radiative heat source, $\dot{\mathrm{q}}_{\mathrm{R}}$, in $\mathrm{W} / \mathrm{m}^{3}$, in the position $\mathrm{x}$ can be computed, respectively, according to:

$$
\begin{aligned}
& \mathrm{q}_{\mathrm{R}}^{\prime \prime}(\mathrm{x})=\sum_{\mathrm{l}=1}^{\mathrm{L}} \int_{\eta} 2 \pi \mu_{1} \omega_{1}\left[\mathrm{I}_{\eta, 1}^{+}(\mathrm{x})-\mathrm{I}_{\eta, 1}^{-}(\mathrm{x})\right] \mathrm{d} \eta \\
& \dot{\mathrm{q}}_{\mathrm{R}}(\mathrm{x})=\sum_{\mathrm{l}=1}^{\mathrm{L}} \int_{\eta}\left\{2 \pi \kappa_{\eta, \mathrm{m}}(\mathrm{x}) \omega_{1}\left[\mathrm{I}_{\eta, 1}^{+}(\mathrm{x})+\mathrm{I}_{\eta, 1}^{-}(\mathrm{x})\right]\right. \\
& \left.-4 \pi \kappa_{\eta, m}(x) \omega_{1} I_{\eta b}(x)\right\} d \eta
\end{aligned}
$$

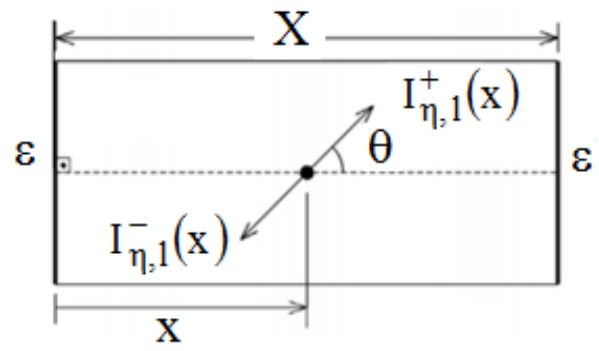

Figure 1. Schematic representation of the 1D-domain.

\section{The WSGG Model}

Proposed by Hottel and Sarofim (1967), the weighted-sum-of-gray-gases (WSGG) model is a global spectral model in which the radiation spectrum is represented by a small set of gray gases with uniform absorption coefficients plus the transparent windows (Modest, 2003). In the WSGG model, the fraction of energy of the blackbody, $a_{j}(T)$, emitted in each region of the spectrum where the pressure absorption coefficient $\left(\kappa_{\mathrm{p} \eta}=\kappa_{\eta} / \mathrm{p}_{\mathrm{a}}\right.$, where $\mathrm{p}_{\mathrm{a}}$ is the partial pressure of the absorbing-emitting species) is $\kappa_{\mathrm{pj}}$, in (atm $\left.\mathrm{m}\right)^{-1}$, can be determined by the polynomial function given below (Smith et al., 1982):

$$
a_{j}(T)=\sum_{k=1}^{K} b_{j, k} T^{k-1}
$$

in which $b_{j, k}$ 's are the polynomial coefficients of the $\mathrm{k}$-th order for the $\mathrm{j}$-th gray gas. For the energy to be conserved, the temperature dependent coefficient for the transparent windows is calculated as $\mathrm{a}_{0}=1-\sum_{\mathrm{j}=1}^{\mathrm{J}} \mathrm{a}_{\mathrm{j}}(\mathrm{T})$. In the present work, it will be used the WSGG coefficients obtained by Dorigon et al., 2013, for a mixture composed of water vapor and carbon dioxide. Besides, for this study, $\mathrm{p}_{\mathrm{a}}=\mathrm{p}_{\mathrm{H} 2 \mathrm{O}}+$ $\mathrm{p}_{\mathrm{CO} 2}$.

Making use of the probabilistic arguments shown in Cassol et al. (2014), and assuming that, in the WSGG model, the gray gases are randomly spread across the spectrum, Fig. 2 shows one spectral hemispherical emissivity distribution of a non-gray wall superimposed with the representation of the pressure absorption coefficient of the $\mathrm{j}$-th gray gas. For every spectral interval, the probability that the blackbody energy to be emitted from the regions where the pressure absorption coefficient is $\kappa_{\mathrm{pj}}$ is given by $a_{j}(T)$. Analogously, the fraction of the blackbody emission of the spectral interval where is the wall emissivity is $\varepsilon_{\mathrm{k}}$ can be interpreted as the probability of the blackbody energy to be emitted in this spectral band. Thus, the joint probability that the emission of the blackbody, at a temperature $\mathrm{T}$, comes from the regions of the spectrum in which the pressure absorption coefficient of the medium is $\kappa_{\mathrm{pj}}$ and the wall emissivity values $\varepsilon_{\mathrm{k}}$ is given by:

$$
a_{j k}(T)=a_{j}(T) \times \frac{\int_{\Delta \eta_{k}} I_{\eta b}(T) d \eta}{I_{b}(T)}
$$

The equation above represents the fraction of the blackbody emissive power that is emitted in the section of the radiation spectrum in which the surface emissivity is $\varepsilon_{\mathrm{k}}$, and can be calculated according to:

$$
\frac{\int_{\Delta \eta_{k}} I_{\eta b}(T) d \eta}{I_{b}(T)}=F_{0 \rightarrow \eta_{k}, T}-F_{0 \rightarrow \eta_{k-1}, T}
$$

where the fraction of blackbody emissive power, $\mathrm{F}_{0 \rightarrow \eta, \mathrm{T}}$, can be calculated from Planck's distribution (Siegel and Howell, 2002; Modest, 2003), and $\eta_{\mathrm{k}}$ and $\eta_{\mathrm{k}-1}$ are, respectively, the upper and lower limits of the spectral band $\Delta \eta_{\mathrm{k}}$.

Regarding to the radiative transfer equation applied to non-gray boundaries, the intensities in the forward and backward directions for the band $\Delta \eta_{k}$, considering only the segments in which the medium pressure absorption coefficient is $\kappa_{\mathrm{pj}}$, are given, respectively, by:

$$
\begin{aligned}
\mu_{1} \frac{d I_{j k, 1}^{+}}{d x}=-\kappa_{p j} p_{a}(x) & I_{j k, 1}^{+}(x) \\
& +\kappa_{p, j} p_{a}(x) a_{j k}(x) I_{b}(x)
\end{aligned}
$$




$$
\begin{aligned}
-\mu_{1} \frac{\mathrm{dI}_{\mathrm{jk}, 1}^{-}}{\mathrm{dx}}=-\kappa_{\mathrm{pj}} \mathrm{p}_{\mathrm{a}}(\mathrm{x}) & \mathrm{I}_{\mathrm{jk}, 1}^{-}(\mathrm{x}) \\
& +\kappa_{\mathrm{p}, \mathrm{j}} \mathrm{p}_{\mathrm{a}}(\mathrm{x}) \mathrm{a}_{\mathrm{jk}}(\mathrm{x}) \mathrm{I}_{\mathrm{b}}(\mathrm{x})
\end{aligned}
$$

Assuming the same hypothesis, the boundary conditions for the WSGG model to the left and to the right of the domain can be expressed, respectively, by:

$$
\begin{aligned}
\mathrm{I}_{\mathrm{jk}, 1}^{+}(\mathrm{x}=0)= & \varepsilon_{\mathrm{k}} \mathrm{a}_{\mathrm{jk}}(\mathrm{x}=0) \mathrm{I}_{\mathrm{b}}(\mathrm{x}=0) \\
& +\frac{\left(1-\varepsilon_{\mathrm{k}}\right)}{\pi} \sum_{\mathrm{l}=1}^{\mathrm{L}} 2 \pi \mu_{1} \omega_{1} \mathrm{I}_{\mathrm{jk}, 1}^{-}(\mathrm{x}=0) \\
\mathrm{I}_{\mathrm{jk}, 1}^{-}(\mathrm{x}=\mathrm{X})= & \varepsilon_{\mathrm{k}} \mathrm{a}_{\mathrm{jk}}(\mathrm{x}=\mathrm{X}) \mathrm{I}_{\mathrm{b}}(\mathrm{x}=\mathrm{X}) \\
& +\frac{\left(1-\varepsilon_{\mathrm{k}}\right)}{\pi} \sum_{\mathrm{l}=1}^{\mathrm{L}} 2 \pi \mu_{1} \omega_{\mathrm{l}} \mathrm{I}_{\mathrm{jk}, \mathrm{l}}^{+}(\mathrm{x}=\mathrm{X})
\end{aligned}
$$

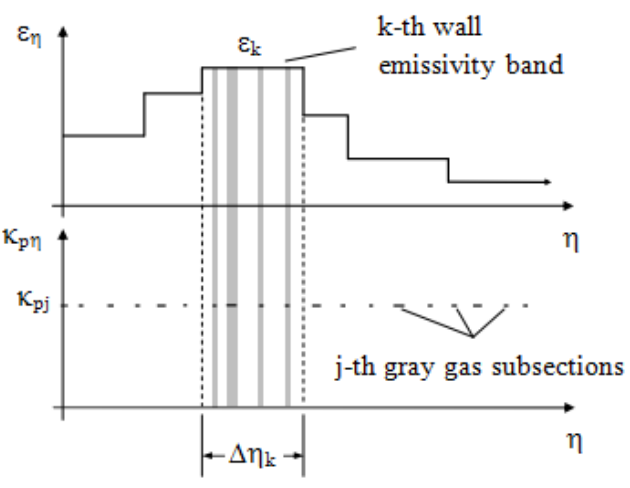

Figure 2. Representation of the regions of spectrum in which the surface emissivity values $\varepsilon_{\mathrm{k}}$ and the absorption coefficient of the medium is $\kappa_{\mathrm{p} j}$.

Finally, the radiative heat flux and the radiative heat source considering the contribution of all bands $\Delta \eta_{\mathrm{k}}$ and all gray gases $\mathrm{j}$ (including the transparent windows) can be determined, respectively, according to the following equations:

$$
\begin{array}{r}
\mathrm{q}_{\mathrm{R}}^{\prime \prime}(\mathrm{x})=\sum_{\mathrm{l}=1}^{\mathrm{L}} \sum_{\mathrm{j}=1}^{\mathrm{J}} \sum_{\mathrm{k}=1}^{\mathrm{K}} 2 \pi \mu_{1} \omega_{1}\left[\mathrm{I}_{j \mathrm{k}, 1}^{+}(\mathrm{x})-\mathrm{I}_{\mathrm{jk}, 1}^{-}(\mathrm{x})\right] \\
\dot{\mathrm{q}}_{\mathrm{R}}(\mathrm{x})=\sum_{\mathrm{l}=1}^{\mathrm{L}} \sum_{\mathrm{j}=1}^{\mathrm{J}} \sum_{\mathrm{k}=1}^{\mathrm{K}} 2 \pi \kappa_{\mathrm{pj}} \mathrm{p}_{\mathrm{a}}(\mathrm{x}) \omega_{1}\left\{\left[\mathrm{I}_{\mathrm{jk}, 1}^{+}(\mathrm{x})+\mathrm{I}_{\mathrm{jk}, 1}^{+}(\mathrm{x})\right]\right. \\
\left.-2 \mathrm{a}_{\mathrm{jk}}(\mathrm{x}) \mathrm{I}_{\mathrm{b}}(\mathrm{x})\right\}
\end{array}
$$

The present study considers a set of cases of non-isothermal and non-homogeneous medium filled by a mixture composed of $\mathrm{H}_{2} \mathrm{O}$ and $\mathrm{CO}_{2}$, with a constant pressure ratio of $\mathrm{p}_{\mathrm{H} 2 \mathrm{O}} / \mathrm{p}_{\mathrm{CO} 2}=2$. The total pressure of the system is $\mathrm{p}=1 \mathrm{~atm}$ and the partial pressures of the participating species are $\mathrm{p}_{\mathrm{H} 2 \mathrm{O}}=0.2$ atm and $\mathrm{p}_{\mathrm{CO} 2}=0.1 \mathrm{~atm}$. For all cases analyzed, the WSGG coefficients, that were obtained from fitting emittance data computed from LBL integration of HITEMP2010 database (Rothman et al., 2010), are presented in Dorigon et al., 2013. The domain consists of two non-gray flat plates, as shown in Fig. 1 , separated by $1 \mathrm{~m}$ of distance; the mesh was divided into 200 equal-sized volumes and the RTE is solved for 30 directions by the discrete ordinates method, using a Gauss-Legendre quadrature. Previous studies show that greater refinement of the domain division did not represent significant variations in the solutions (Dorigon et al., 2013; Fonseca, 2017). According to Ziemniczak et al. (2013), four gray gases are sufficient to perform the present analysis, so that, as shown in the table below, this study was carried out with $\mathrm{j}=4$.

\section{RESULTS AND DISCUSSION}

To evaluate the accuracy of the methodology developed to apply the WSGG model to media bounded by non-gray walls, the solutions are compared against the LBL integration. The following temperature profiles, in $\mathrm{K}$, are considered:

$$
\begin{gathered}
T(x)=400+1400 \sin ^{2}(2 \pi x) \\
T(x)=\left\{\begin{array}{l}
880+920 \sin ^{2}(2 \pi x), \text { if } x \leq 0.25 \mathrm{~m} \\
400+1400\left\{1-\sin ^{3 / 2}\left[\frac{2}{3} \pi(x-0.25)\right]\right\}, \\
\text { if } x>0.25 \mathrm{~m}
\end{array}\right.
\end{gathered}
$$

As with the temperature, two molar concentrations of $\mathrm{CO}_{2}$ are considered:

$$
\begin{gathered}
\mathrm{Y}_{\mathrm{CO} 2}(\mathrm{x})=0.2 \sin ^{2}(2 \pi \mathrm{x}) \\
\mathrm{Y}_{\mathrm{CO} 2}(\mathrm{x})=\left\{\begin{array}{l}
0.25 \sin ^{2}(2 \pi \mathrm{x}), \text { if } \mathrm{x} \leq 0.25 \mathrm{~m} \\
0.25\left\{1-\sin \left[\frac{2}{3} \pi(\mathrm{x}-0.25)\right]\right\}, \\
\text { if } \mathrm{x}>0.25 \mathrm{~m}
\end{array}\right.
\end{gathered}
$$

For water vapor, the molar concentration is the double of carbon dioxide $\left(\mathrm{Y}_{\mathrm{H} 2 \mathrm{O}}=2 \mathrm{Y}_{\mathrm{CO} 2}\right)$. Figures 3 and 4 show the temperature and concentration profiles, respectively. To generate the results showed in the next sections, Eqs. (18) and (20) were employed together, since these expressions present behavior with double symmetry (posteriorly referenced as D.S. profile); similarly, Eqs. (19) and (21) also were combined, since these equations do not present symmetry (posteriorly referenced as N.S. profile). The partial pressure of the absorbingemitting species can be determined as $\mathrm{p}_{\mathrm{a}}(\mathrm{x})=$ 
$\left[\mathrm{Y}_{\mathrm{H} 2 \mathrm{O}}(\mathrm{x})+\mathrm{Y}_{\mathrm{CO} 2}(\mathrm{x})\right] p$, in which the ratio $\mathrm{Y}_{\mathrm{H} 2 \mathrm{O}} / \mathrm{Y}_{\mathrm{CO} 2}$ is kept equal to 2 .

The accuracy of the methodology applied is computed by means of the relative deviation between the radiative heat fluxes and the radiative heat sources obtained with the WSGG methods and the LBL solution:

$$
\begin{aligned}
& \delta=\left|\frac{\mathrm{q}_{\mathrm{R}, \mathrm{WSGG}}^{\prime \prime}-\mathrm{q}_{\mathrm{R}, \mathrm{LBL}}^{\prime \prime}}{\max \left(\mathrm{q}_{\mathrm{R}, \mathrm{LBL}}^{\prime}\right)}\right| \times 100 \% \\
& \zeta=\left|\frac{\dot{\mathrm{q}}_{\mathrm{R}, \mathrm{WSGG}}-\dot{\mathrm{q}}_{\mathrm{R}, \mathrm{LBL}}}{\max \left(\dot{\mathrm{q}}_{\mathrm{R}, \mathrm{LBL}}\right)}\right| \times 100 \%
\end{aligned}
$$

where $\delta$ and $\zeta$ are the local deviations of the radiative heat flux and the radiative heat source, respectively; and $\max \left(\mathrm{q}_{\mathrm{R}, \mathrm{LBL}}^{\prime \prime}\right)$ and $\max \left(\dot{\mathrm{q}}_{\mathrm{R}, \mathrm{LBL}}\right)$ are, respectively, the maximum absolute values of the radiative heat flux and the radiative heat source obtained by the LBL integration in each case analyzed. Two deviations will be of interest for the analysis performed in this paper: the maximum $\left(\delta_{\max }\right.$ and $\left.\zeta_{\max }\right)$ and the average $\left(\delta_{\text {avg }}\right.$ and $\left.\zeta_{\text {avg }}\right)$ deviations over the domain.

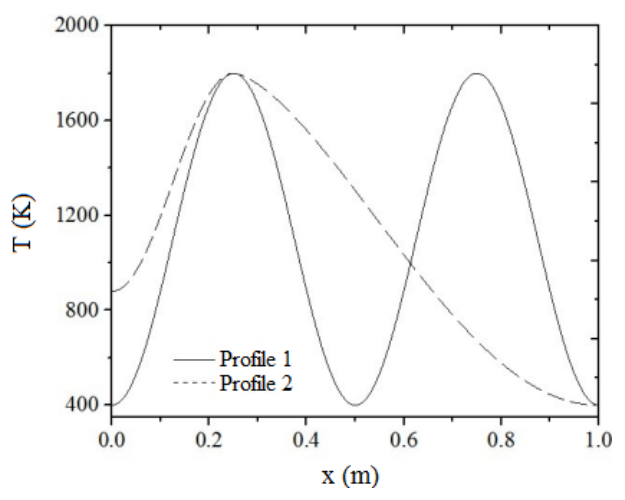

Figure 3. Temperature profiles.

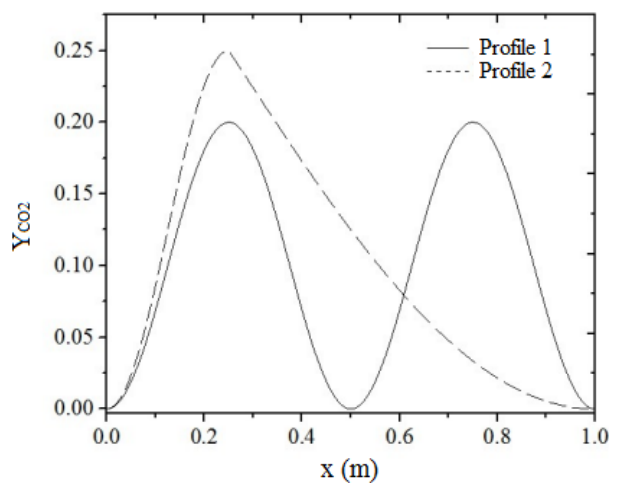

Figure 4. $\mathrm{CO}_{2}$ molar concentration profiles.

In the next sections, the results presented consider the problem illustrated in Fig. 1 and, when the surfaces that bound the medium are assumed as non-gray, their spectral emissivity is described as a combination of spectral intervals, where to each interval is associated a constant emissivity value, as given in Fig. 5. It will be two spectral intervals: one consisting of three bands and the other formed of five bands. Conversely, for the gray walls, the constant value adopted for the emissivity are computed as an average weighted by the blackbody energy fraction of the spectral intervals that compose the non-gray emissivities's profiles. Solutions considering black boundaries also are presented, and these results are already known in the literature. For simplicity, the two surfaces that bound the medium are considered to have the same emissivity. The gray surface total emissivity, $\varepsilon$, is determined by means of a ratio between the spectral emissivity and the Planck's blackbody distribution function at the wall temperature, $\mathrm{T}_{\mathrm{w}}$, according to:

$$
\varepsilon=\frac{\int_{\eta} \varepsilon_{\eta} I_{\eta_{b}}\left(T_{w}\right) d \eta}{I_{b}\left(T_{w}\right)}
$$

Considering the gray assumption, the wall absorptivity is equal to the emissivity wall $(\alpha=\varepsilon)$. For the black wall case, $\alpha=\varepsilon=1$. In the next sections, it will be given the values of the equivalent emissivities $\bar{\varepsilon}$ calculated for each spectral distribution considered: this value was used in the calculation of the solution with gray boundaries. Moreover, in this paper, it was chosen to present the spectral distributions of the walls' emissivities in terms of the wavelength $\lambda(\lambda=1 / \eta)$, instead of the wavenumber $\eta$, as can be observed in Fig. 5 .

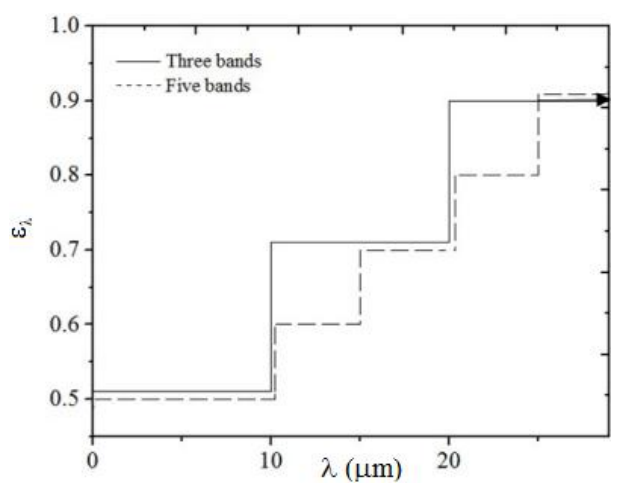

Figure 5. Surface emissivities with three-band and five-band stepwise variations.

\section{Three Spectral Intervals}

For the case with three bands, it is considered the following spectral intervals for the wall's emissivities: $\varepsilon=0.5$ for $\lambda \leq 10 \mu \mathrm{m} ; \varepsilon=0.7$ for $10 \mu \mathrm{m}$ $<\lambda \leq 20 \mu \mathrm{m}$; and $\varepsilon=0.9$ for $\lambda>20 \mu \mathrm{m}$, as illustrated in Fig. 5. Moreover, the medium is considered to be non-isothermal, varying according to the profile 
temperatures of the Eqs. (18) and (19), and nonhomogeneous, varying with the molar concentration profiles expressed in Eqs. (20) and (21). It is assumed that the medium is formed by a mixture of $\mathrm{H}_{2} \mathrm{O}$ and $\mathrm{CO}_{2}$, with a constant pressure ratio of $\mathrm{p}_{\mathrm{H} 2 \mathrm{O}} / \mathrm{p}_{\mathrm{CO} 2}=2$, using, therefore, the WSGG coefficients presented in Dorigon et al., 2013.

Combining the double symmetrical profiles (designated by D.S. profile in Tab. 1), i.e. Eq. (18) for the temperature and Eq. (20) for the molar concentration, Figs. 6 and 7 show the results obtained for the radiative heat flux, $\mathrm{q}_{\mathrm{R}}$, and the radiative heat source, $\dot{\mathrm{q}}_{\mathrm{R}}=\left(-\mathrm{dq}_{\mathrm{R}}^{\prime \prime} / \mathrm{dx}\right)$, respectively, assuming nongray, gray and black surfaces. As can be seen in Fig. 6 , the methodology developed in this work, represented by the WSGG non-gray solution, presents a good agreement with the LBL integration throughout the domain, since, according to Tab. 1, the maximum deviation between these two approaches is $2.40 \%$ and the average deviation is $1.03 \%$. Besides, Fig. 6 also shows the results for gray and black boundaries, respectively WSGG gray (with $\bar{\varepsilon}_{\text {left }}=\bar{\varepsilon}_{\text {right }}=0.633$ ) and WSGG black solutions. In Tab. 1, comparison 1 indicates the LBL non-gray vs. WSGG non-gray; comparison 2 indicates LBL non-gray vs. WSGG gray; and comparison 3 indicates LBL non-gray vs. WSGG black, and the same references are applied in Tab. 2, that will appear later. It can be perceived that, in the regions close to the domain walls, the deviations in relation to the LBL benchmark solution of the gray and black curves become more important, reaching maximum values of $10.96 \%$ (WSGG gray) and $41.80 \%$ (WSGG black). Figure 7 presents the radiative heat source for all solutions, where it is observed that the deviations in relation to the LBL integration of the WSGG model in the three approaches are in the regions close to the walls in which the medium temperature is higher. Analogously to that seen in Fig. 6, the WSGG nongray solution has a good match with the LBL method, once the maximum and average deviations are $3.12 \%$ and $1.06 \%$, respectively, as shown in Tab. 1. Comparing with the WSGG gray solution, the maximum deviation found is $5.14 \%$; when confronted with the WSGG black solution, the maximum deviation is $15.08 \%$.

Joining the not symmetrical profiles (designated by N.S. profile in Tab. 1), i.e. Eq. (19) for the temperature and Eq. (21) for the molar concentration, Figs. 8 and 9 present, respectively, $\mathrm{q}_{\mathrm{R}}^{\prime \prime}$ and $\dot{\mathrm{q}}_{\mathrm{R}}$, for non-gray, gray and black walls. Figure 8 shows a satisfactory concordance between the radiative heat flux found by the WSGG non-gray solution and the LBL integration, seeing that, as presented in Tab. 1, the maximum and the average deviations for the radiative heat flux are, approximately, $3 \%$ and $1 \%$, respectively.

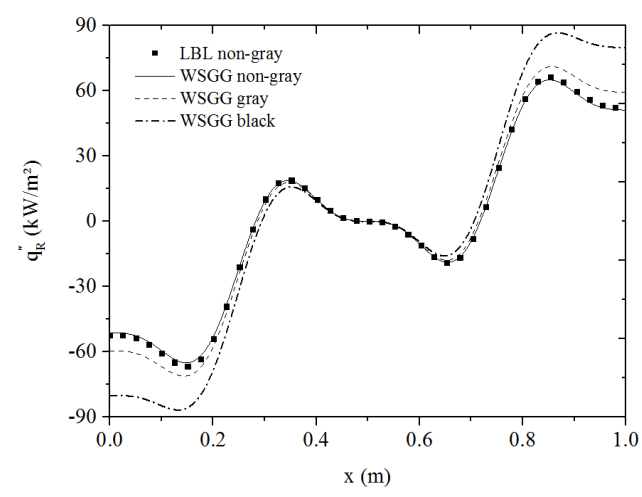

Figure 6. Radiative heat flux considering three spectral intervals and double symmetrical profiles.

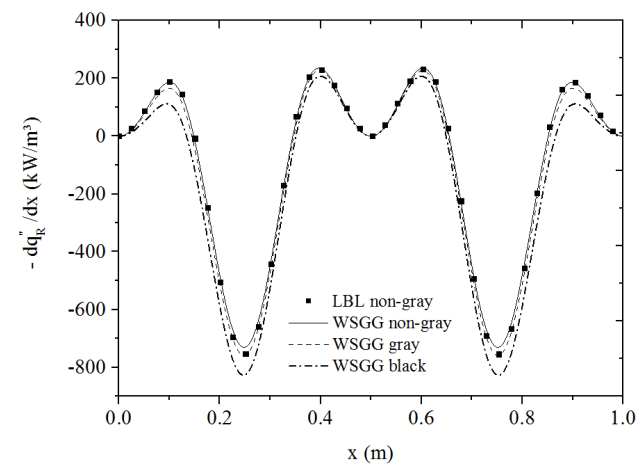

Figure 7. Radiative heat source considering three spectral intervals and double symmetrical profiles.

Table 1. Percentage average and maximum deviations for $\mathrm{q}_{\mathrm{R}}$ and $\dot{\mathrm{q}}_{\mathrm{R}}$, comparing the WSGG models against the solution with LBL non-gray for three spectral intervals.

\begin{tabular}{|c|c|c|c|c|c|}
\hline Profile & Comparison & $\begin{array}{c}\delta_{\max } \\
(\%)\end{array}$ & $\begin{array}{c}\delta_{\text {avg }} \\
(\%)\end{array}$ & $\begin{array}{c}\zeta_{\max } \\
(\%)\end{array}$ & $\begin{array}{c}\zeta_{\text {avg }} \\
(\%)\end{array}$ \\
\hline \multirow{3}{*}{ D.S. } & 1 & 2.40 & 1.03 & 3.12 & 1.06 \\
\cline { 2 - 6 } & 2 & 10.96 & 4.65 & 5.14 & 1.93 \\
\cline { 2 - 6 } & 3 & 41.80 & 17.98 & 15.08 & 7.37 \\
\hline \multirow{3}{*}{ N.S. } & 1 & 2.78 & 1.12 & 2.19 & 0.53 \\
\cline { 2 - 6 } & 2 & 11.41 & 3.88 & 4.36 & 1.08 \\
\cline { 2 - 6 } & 3 & 44.92 & 27.71 & 15.62 & 6.59 \\
\hline
\end{tabular}

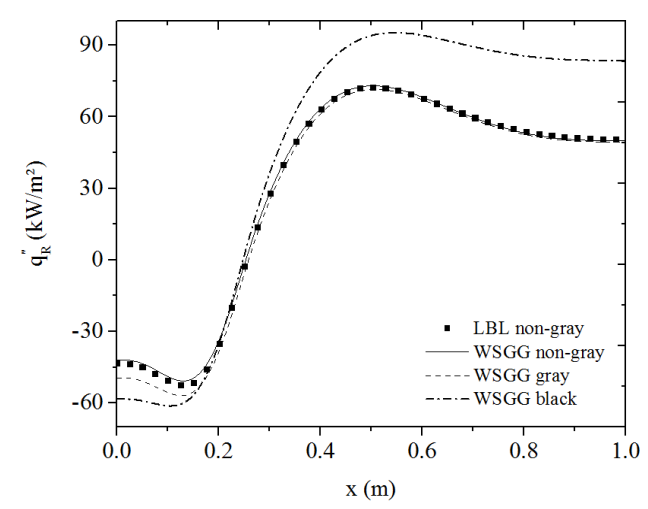

Figure 8. Radiative heat flux considering three spectral intervals and not symmetrical profiles. 
Regarding to the results for gray and black surfaces, also shown in Fig. 8, the WSGG gray solution (where $\bar{\varepsilon}_{\text {left }}=0.527$ and $\bar{\varepsilon}_{\text {right }}=0.633$, since $\mathrm{T}_{\text {left }}=880 \mathrm{~K}$ and $\mathrm{T}_{\text {right }}=400 \mathrm{~K}$ ) presents a good agreement over almost the entire domain, except close to the left boundary, where the deviation between the two approaches is greater, reaching $11 \%$ when compared to the benchmark solution; assuming the wall as black, the maximum deviation can reach approximately $45 \%$, with an average deviation of around 30\%. The radiative heat source, Fig. 9, presents little discrepancies for solutions with nongray and gray walls, with maximum values of $2.19 \%$ and $4.26 \%$, respectively. Although with a smaller order of magnitude than the radiative heat flux considering black surfaces (which was almost 45\%), $\dot{\mathrm{q}}_{\mathrm{R}}$, for this approach, shows a maximum deviation of about $16 \%$, according to Tab. 1 .

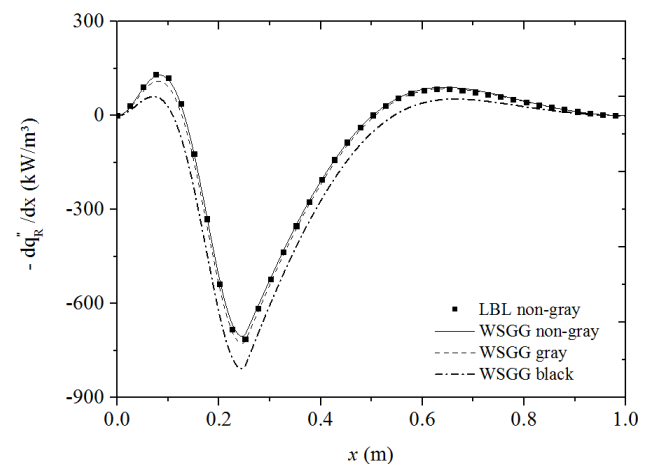

Figure 9. Radiative heat source considering three spectral intervals and not symmetrical profiles.

\section{Five spectral intervals}

Solving the problem considering five bands, it is used the following spectral distribution: $\varepsilon=0.5$ for $\lambda$ $\leq 10 \mu \mathrm{m} ; \varepsilon=0.6$ for $10 \mu \mathrm{m}<\lambda \leq 15 \mu \mathrm{m} ; \varepsilon=0.7$ for $15 \mu \mathrm{m}<\lambda \leq 20 \mu \mathrm{m} ; \varepsilon=0.8$ for $20 \mu \mathrm{m}<\lambda \leq 25 \mu \mathrm{m}$; and $\varepsilon=0.9$ for $\lambda>25 \mu \mathrm{m}$, as shown in Fig. 5 .

Assuming the double symmetrical profiles, Eqs. (18) and (20), Fig. 10 presents the radiative heat flux for non-gray, gray and black walls. Based on Tab. 2 and Fig. 10, the WSGG model applied to non-gray surfaces agrees very well with the LBL benchmark solution, since the maximum deviation between the two approaches is $2.30 \%$ and the average deviation is less than $1 \%$. Although similar to the results presented in the previous section, for the case with double symmetry and three spectral intervals, the deviations between the two solutions considering non-gray boundaries are even smaller, even if the magnitude of $q_{R}$ does not seem to change significantly. This fact indicates that the solution methodology applied to the problem studied in this work is not very sensitive to the number of regions in which the spectrum is divided. Regarding to the methodology applied to gray $(\bar{\varepsilon}=0.601)$ and black surfaces, the maximum deviations obtained are, respectively, $8.27 \%$ and $42.16 \%$ in relation to the LBL integration. For the radiative heat source, Fig. 11, the maximum and average deviations found between the two methods are also smaller than those found in Fig. 7, corresponding to values of $3 \%$ and $1 \%$, respectively. For gray and black walls, the maximum deviations are $4.27 \%$ and $15.09 \%$, respectively, when compared with $\dot{\mathrm{q}}_{\mathrm{R}}$ obtained with the benchmark solution.

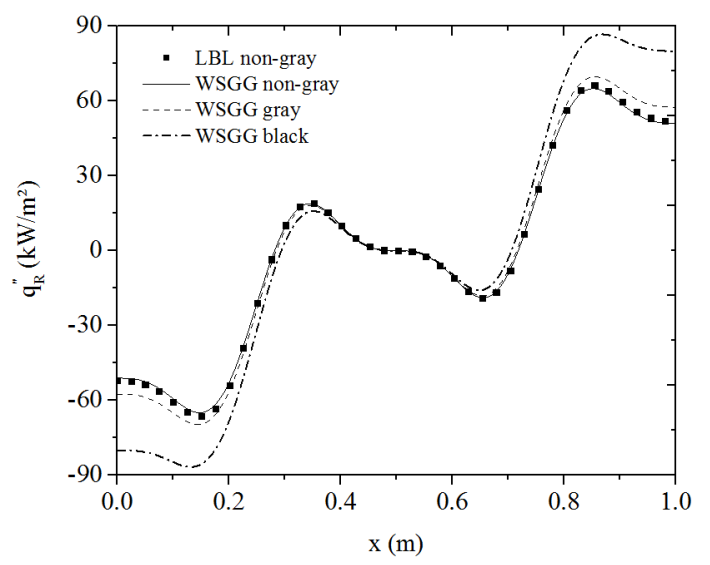

Figure 10. Radiative heat flux considering five spectral intervals and double symmetrical profiles.

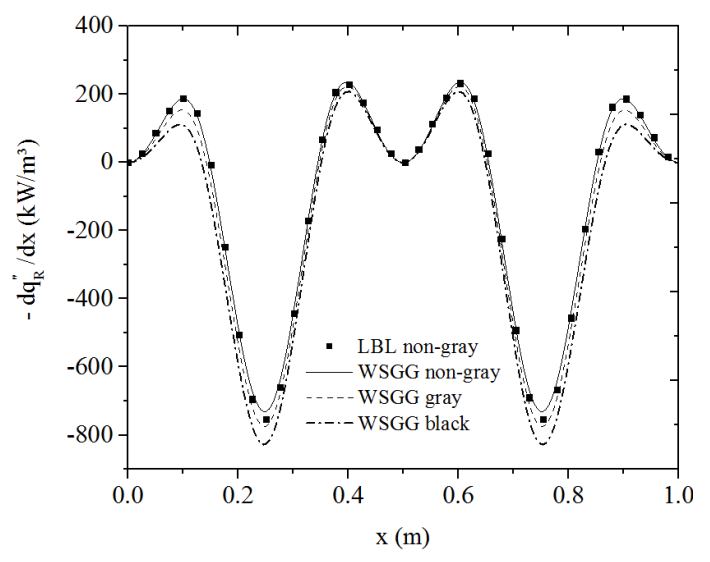

Figure 11. Radiative heat source considering five spectral intervals and double symmetrical profiles.

Table 2. Percentage average and maximum deviations for $\mathrm{q}_{\mathrm{R}}$ and $\dot{\mathrm{q}}_{\mathrm{R}}$, comparing the WSGG models against the solution with LBL non-gray for five spectral intervals.

\begin{tabular}{|c|c|c|c|c|c|}
\hline Profile & Comparison & $\begin{array}{c}\delta_{\max } \\
(\%)\end{array}$ & $\begin{array}{c}\delta_{\text {avg }} \\
(\%)\end{array}$ & $\begin{array}{c}\zeta_{\max } \\
(\%)\end{array}$ & $\begin{array}{c}\zeta_{\text {avg }} \\
(\%)\end{array}$ \\
\hline \multirow{3}{*}{ D.S. } & 1 & 2.30 & 0.99 & 3.03 & 1.04 \\
\cline { 2 - 6 } & 2 & 8.27 & 3.48 & 4.27 & 1.47 \\
\cline { 2 - 6 } & 3 & 42.16 & 18.15 & 15.09 & 7.39 \\
\hline \multirow{3}{*}{ N.S. } & 1 & 2.63 & 1.08 & 2.11 & 0.52 \\
\cline { 2 - 6 } & 2 & 8.51 & 2.96 & 3.56 & 0.84 \\
\cline { 2 - 6 } & 3 & 45.43 & 28.03 & 15.71 & 6.63 \\
\hline
\end{tabular}


Figures 12 and 13 show the radiative heat flux and the radiative heat source, respectively, considering the not symmetrical profiles, Eqs. (19) and (21). According to Fig. 12, there is a good agreement between the results for the heat flux obtained with the WSGG model applied to non-gray walls and the LBL integration, because, as can be seen in Tab. 2, the maximum and the average deviations are, respectively, $2.63 \%$ and $1.08 \%$. For gray $\left(\bar{\varepsilon}_{\text {left }}=0.519\right.$ and $\left.\bar{\varepsilon}_{\text {right }}=0.601\right)$ and black boundaries, the maximum deviations found are $8.51 \%$ (WSGG gray) and $45.43 \%$ (WSGG black). Finally, Fig. 13 and Tab. 2 show the following maximum deviations in comparison with the LBL solution: $2.11 \%$ (WSGG non-gray), 3.56\% (WSGG gray) and $15.71 \%$ (WSGG black).

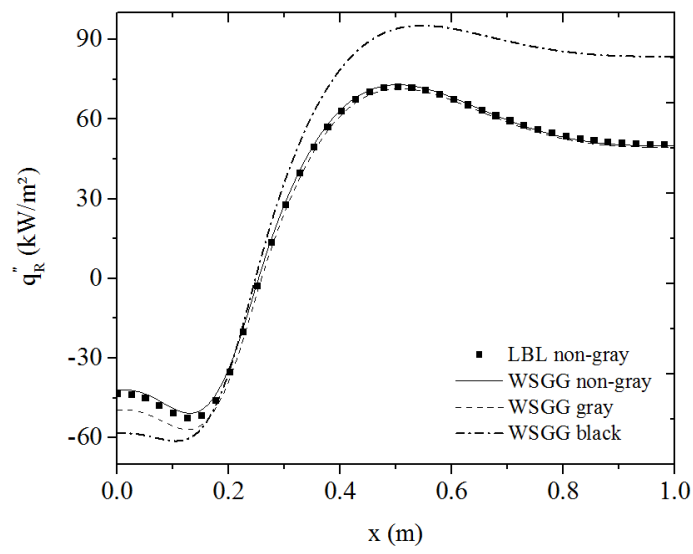

Figure 12. Radiative heat flux considering five spectral intervals and not symmetrical profiles.

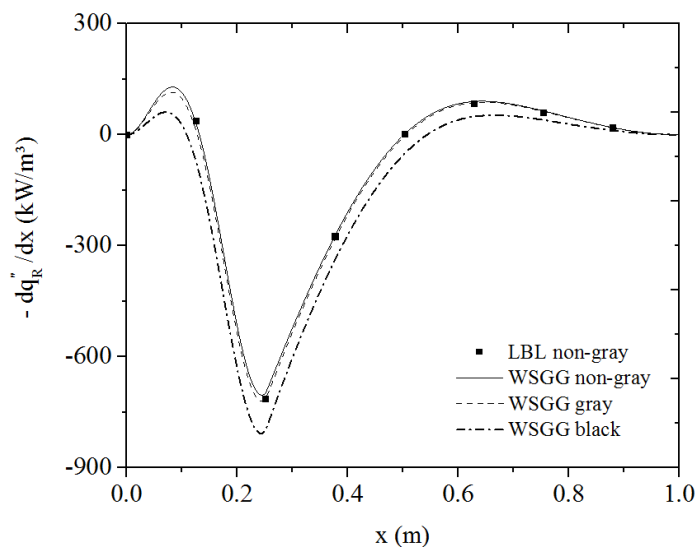

Figure 13. Radiative heat source considering five spectral intervals and not symmetrical profiles.

\section{CONCLUSIONS}

In the present study, it was analyzed the radiative heat transfer through the application of the WSGG model to a one-dimensional, non-isothermal and non-homogeneous slab composed of a mixture of water vapor and carbon dioxide, bounded by nongray walls. The accuracy of the developed methodology (WSGG non-gray solution) was evaluated by means of comparisons with the LBL benchmark solution.

From the point of view of the simplicity of the WSGG model, the solution methodology is able to satisfactorily predict the behavior of the radiative heat flux and the radiative heat source when compared to the results obtained by the LBL integration applied to non-gray surfaces, since the maximum deviations found between the two approaches, for all cases investigated, did not exceed $3 \%$. However, it was verified that the approximation in assuming the walls as gray or black can lead to not negligible deviations between the solutions, reaching values above $40 \%$

\section{ACKNOWLEDGEMENTS}

The second author thanks CNPq (Brazil) for research grants 309961/2013-0 and 476490/2013-8.

\section{REFERENCES}

Brittes, R., Centeno, F. R., Ziemniczak, A., and França, F. H. R., 2017, WSGG Model Correlations to Compute Non-Gray Radiation from Carbon Monoxide in Combustion Applications, ASME Journal of Heat Transfer, Vol. 139, No. 4, pp. 041202-1-041202-7.

Cassol, F., Brittes, R., França, F. H. R., and Ezekoye, O. A., 2014, Application of the WeightedSum-of-Gray-Gases Model for Media Composed of Arbitrary Concentrations of $\mathrm{H}_{2} \mathrm{O}, \mathrm{CO}_{2}$ and Soot, International Journal of Heat Mass Transfer, Vol. 79, pp. 796-806.

Fonseca, R. J. C., Ziemniczak, A., and França, F. H. R., 2015, Application of the Weighted-Sum-ofGray-Gases Model in Enclosures with Gray Surfaces, in: 23rd International Congress of Mechanical Engineering, Rio de Janeiro, Brazil.

Fonseca, R. J. C., and França, F. H. R., 2016, Application of the Weighted-Sum-of-Gray-Gases Model in Media Bounded by Non-Gray Walls, in: 16th Brazilian Congress of Thermal Sciences and Engineering, Vitória, Brazil.

Fonseca, R. J. C., 2017, Aplicação do Modelo da Soma-Ponderada-de-Gases-Cinza a Sistemas com Superfícies não Cinzas, Master Thesis, UFRGS, Porto Alegre, RS. (in Portuguese)

Hottel, H. C., and Sarofim, A. F., 1967, Radiation Transfer, McGraw-Hill.

Modest, M. F., 2003, Radiative Heat Transfer, Academic Press.

Rothman, L. S., Gordon, I. E., Barber, R. J., Dothe, H., Gamache, R. R., Goldman, A., Perevalov, V. I., Tashkun, S. A., and Tennyson, J., 2010, HITEMP, The High-Temperature Molecular Spectroscopic Database, Journal of Quantitative Spectroscopy and Radiative Transfer, Vol. 111, pp. 2139-2150. 
Siegel, R., and Howell, J., 2002, Thermal Radiation Heat Transfer, Taylor and Francis.

Smith, T. F., Shen, Z. F., and Friedman, J. N., 1982, Evaluation of Coefficients for the Weighted Sum of Gray Gases Model, Journal of Heat Transfer, Vol. 104, pp. 602-608.

Solovjov, V. P., Lemonnier, D., and Webb, B. W., 2013, Efficient Cumulative Wavenumber Model of Radiative Transfer in Gaseous Media Bounded by Non-Gray Walls, Journal of Quantitative Spectroscopy and Radiative Transfer, Vol. 128, pp. $2-9$.

Ziemniczak, A., Brittes, R., Cassol, F., and França, F. H. R., 2013, Evaluation of the Number of Gray Gases in the WSGG Model, in: 22nd International Congress of Mechanical Engineering, Ribeirão Preto, Brazil. 